\title{
El Periodismo de Salud en España. La información de salud en la prensa española (2000-2010): aproximación a los estudios documentales existentes
}

\author{
Milagros RonCO LÓPEZ \\ milagros.ronco@ehu.es \\ Profesora. Departamento de Periodismo \\ Universidad del País Vasco UPV/EHU \\ ORCID.- 0000-0002-4571-0845 \\ Carmen PEÑAFIEL \\ Universidad del País Vasco / Euskal Herriko Unibertsitatea \\ carmen.penafiel@ehu.es \\ Lázaro ECHEGARAY \\ Escuela Universitaria de la Cámara de Comercio de Bilbao, EUCCB - \\ lazaro.echegaray@euccb.com ,
}

Recibido: $13 / 04 / 2014$

Aceptado: 22/05/2014

\section{RESUMEN}

Está demostrada la importancia que nuestra sociedad le concede a la salud. Las diversas encuestas de los diversos organismos competentes así como múltiples investigaciones sociales, colocan el tema de la salud en un lugar prioritario entre los intereses de la población en España. En este trabajo, hacemos un análisis de los principales estudios llevados a cabo tanto por organizaciones internacionales -la OMS- como nacionales -Observatorio de la Comunicación Científica, Fundación Vilá Casas, Instituto de Comunicación InCom de la UAB, Instituto Internacional de Comunicación y Salud INICyS, Seminario de Comunicación y Salud de la Universidad de Navarra y los Grupos de Trabajo sobre Comunicación y Salud de la SEMFyC-. Por último, hacemos un recorrido en torno a los principales estudios realizados por investigadores diversos

Palabras clave: Estudios sobre salud, Documentación sobre salud, Comunicación y salud, Periodismo científico

The health information in Spanish press (2000-2010): approach to the existing documentary studies

\begin{abstract}
The importance which our society places on health has been clearly shown. The surveys from diverse competent organizations, as well as multiple social research projects have positioned the health topic as a major priority among the population's interests in Spain and this interest has been reflected in the mass media. In this paper, we analyze the main investigations and studies carried out by international authorities-WHO- as well as national organizations-Scientific Communication Observatory, Vilá
\end{abstract}


Casas Foundation, Communication Institute (InCom-UAB), International Institute of Communication and Health (INICyS), Communication and Health Seminar of the University of Navarra and the Work Teams on Communication and Health of the Spanish Society for Family and Community Medicine (SEMFyC)-. Finally, we take a review around the principal studies carried out by different researchers

Keywords: Studies on health, Documentation on health, Communication and health, Scientific journalism.

\section{INTRODUCCIÓN}

Abordamos este tema con la intención de hacer un estudio en torno a la investigación y al análisis dedicados al Periodismo de Salud en la España contemporánea ${ }^{1}$. Para ello hemos recabado sus antecedentes más directos, hemos analizado el papel de las Organizaciones Internacionales, hemos recogido las principales investigaciones sobre el particular, hemos incorporado las descripciones (resúmenes y valoraciones) de libros y artículos seleccionados que tratan el tema incluyendo, por último, el análisis de las principales Asociaciones Españolas de Periodismo Científico. Todo ello en función de la importancia que nuestra sociedad le concede a la salud y a la calidad de vida.

Año tras año, las diversas encuestas de los diversos organismos competentes así como múltiples investigaciones sociales, colocan el tema de la salud en el lugar prioritario entre los intereses de la población. La muestra comparativa procedente del Barómetro Sanitario del Ministerio de Sanidad (2005-2010) recogida en abril de 2011 y completada en enero del 2012, refleja la preocupación por la salud por parte de la sociedad a nivel nacional.

${ }^{1}$ Investigación subvencionada por la Universidad del país Vasco UPV/EHU sobre "La información de salud en la prensa diaria vasca (2000-2010)", Código EHU/10/47. El equipo está compuesto por: IP: Idoia Camacho Markina. Milagros Ronco López, Carmen Peñafiel Saiz, Alazne Aiestaran Yarza profesoras del Departamento de Periodismo de la UPV/EHU; y Lázaro Echegaray Eizaguirre, investigador y profesor del Centro colaborador Escuela Universitaria de la Cámara de Comercio de Bilbao. 
Tabla 1. Áreas de interés para la ciudadanía ${ }^{2}$ ¿Cuál de las siguientes áreas considera de mayor interés para los ciudadanos?

\begin{tabular}{lcccccc}
\hline & 2005 & 2006 & 2007 & 2008 & 2009 & $\mathbf{2 0 1 0}$ \\
\hline Defensa & 0,8 & 1,2 & 1,0 & 0,9 & 1,1 & $\mathbf{1 , 1}$ \\
\hline Educación & 19,5 & 18,8 & 20,1 & 20,2 & 21,1 & $\mathbf{2 2 , 7}$ \\
\hline Sanidad & $\mathbf{3 0 , 3}$ & $\mathbf{2 8 , 3}$ & $\mathbf{2 8 , 3}$ & $\mathbf{2 8 , 1}$ & $\mathbf{2 9}$ & $\mathbf{2 9 , 2}$ \\
\hline Vivienda & 20,1 & 20,1 & 20,3 & 20,3 & 17,2 & $\mathbf{1 3 , 5}$ \\
\hline Pensiones & 10,4 & 9,7 & 10,1 & 11,6 & 11,3 & $\mathbf{1 4 , 1}$ \\
\hline Transportes & 0,6 & 0,7 & 0,9 & 0,7 & 0,8 & $\mathbf{0 , 8}$ \\
\hline Seguridad ciudadana & 10,0 & 13,1 & 11,7 & 10,1 & 9,7 & $\mathbf{7 , 8}$ \\
\hline Otras áreas Servicios Sociales & 3,6 & 4,1 & 3,5 & 4,1 & 5,4 & $\mathbf{5 , 7}$ \\
\hline NS-NC & 4,7 & 4,0 & 4,1 & 4,1 & 4,3 & $\mathbf{5 , 2}$ \\
\hline
\end{tabular}

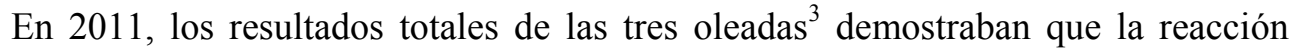
social continuaba en la misma línea y en alza:

Tabla 2. Interés Ciudadano (CIS)

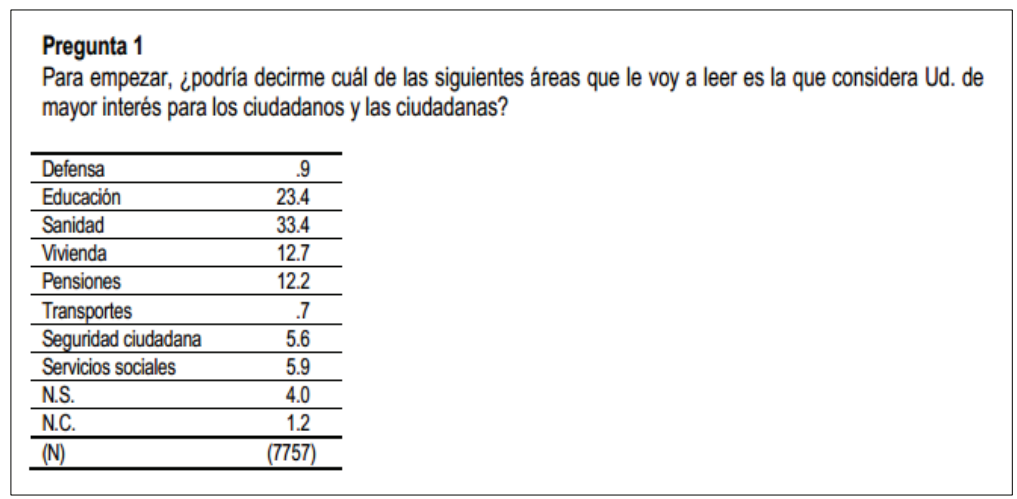

${ }^{2}$ Barómetro Sanitario, 2010 Instituto de Información Sanitaria, 2011, p. 44

http://www.msps.es/estadEstudios/estadisticas/inforRecopilacionens/barometro/home.htm

${ }^{3}$ CIS. Barómetro Sanitario. Estudios no 2862, 2906 y 2916 febrero-octubre 2011. Pregunta 1, p. 1.

http://www.msc.es/estadEstudios/estadisticas/docs/BS_2011_total_marginales.pdf 
Tabla 3. Las últimas encuestas, realizadas en 2012 (marzo-noviembre) y publicadas en 2013 , reiteran ese interés: ${ }^{4}$
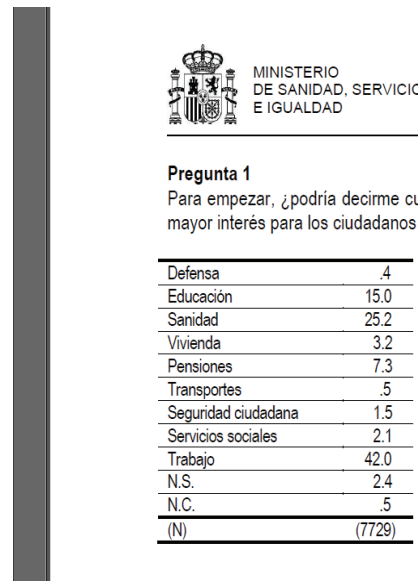

CIS

Pregunta 1

Para empezar, ¿podría decirme cuál de las siguientes áreas que le voy a leer es la que considera Ud. de mayor interés para los ciudadanos y las ciudadanas?

\begin{tabular}{lr}
\hline Defensa & .4 \\
\hline Educación & 15.0 \\
\hline Sanidad & 25.2 \\
\hline Vivienda & 3.2 \\
\hline Pensiones & 7.3 \\
\hline Transportes & .5 \\
\hline Seguridad ciudadana & 1.5 \\
\hline Servicios sociales & 2.1 \\
\hline Trabajo & 42.0 \\
\hline N.S. & 2.4 \\
\hline N.C. & .5 \\
\hline (N) & $(7729)$ \\
\hline
\end{tabular}

Tabla 4. Porcentaje de interés de los ciudadanos por la Sanidad (1995-2012)

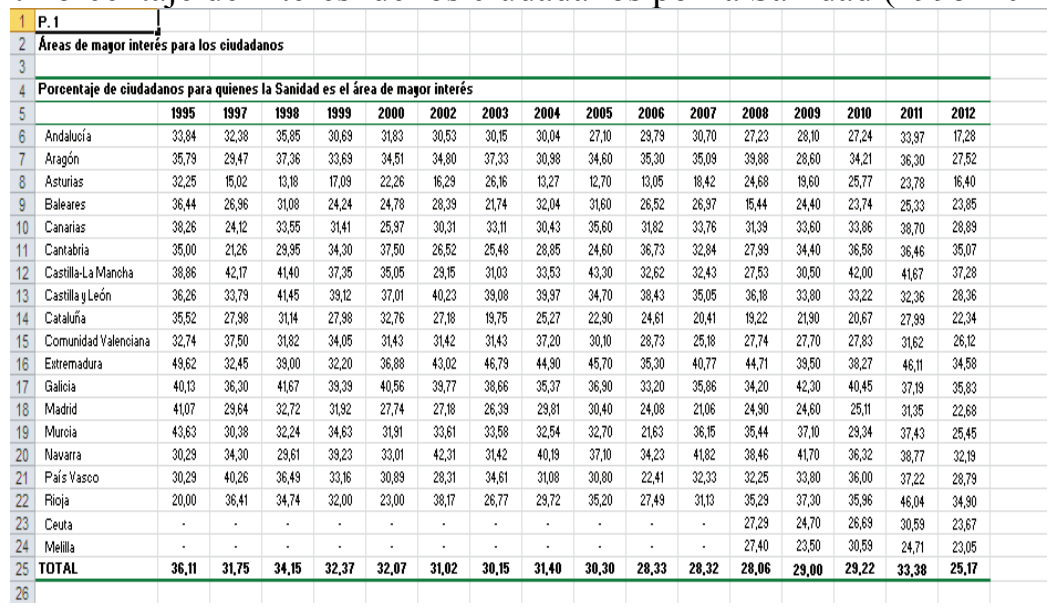

\section{ANTECEDENTES}

Como demuestran las encuestas periódicamente, la salud es una de las principales preocupaciones de la sociedad. Pero es una inquietud que no hace tanto tiempo que se ve reflejada en los medios de comunicación del Estado español

4

https://www.msssi.gob.es/estadEstudios/estadisticas/sisInfSanSNS/informeAnual2012.htm (tablas 3 y 4) Consultado: 12-9-2013 
En 1977, el presidente Adolfo Suárez crea el Ministerio de Sanidad y Seguridad Social para impulsar la reforma sanitaria como forma de modernización del país. La prensa escrita se encuentra sin periodistas especializados y sin una idea clara de dónde ubicar la incipiente información de la política sanitaria

"Es esta la razón por la cual durante toda la década de los setenta, la información sobre política sanitaria se ubica en diferentes secciones de los periódicos: nacional, medicina y ciencia, local, etc. (...) Ante la avalancha de informaciones referidas a la reforma sanitaria, los medios improvisan: unas veces aparece su referencia en nacional y otras en medicina y ciencia. Pero la respuesta es improvisada. A finales de los setenta y principios de los ochenta, la prensa escrita inicia un camino: la creación de la sección de Sociedad, equiparable a Nacional, Local o Economía. En ella se ubican muchas de las informaciones que afectan a los ciudadanos: la educación, la sanidad, los problemas de la justicia, los sucesos, los avances médicos.

Los años ochenta y buena parte de los noventa suponen una consolidación de este formato. Sociedad gana terreno aunque no llegue a las tasas de importancia de Economía, Nacional o Internacional. En los noventa, la información sobre política

sanitaria se enquista al fallar la reforma que proponía el Informe Abril $^{5}(\ldots)$ los medios terminan por desinteresarse por las noticias relacionadas con el sistema sanitario y su incumplida reforma, para empezar a mirar de forma más preferente por todo aquello que interesa a los ciudadanos: sus hábitos alimenticios, la salud de sus hijos, la prevención de las enfermedades, los consejos de salud" (Jurado. 2003, p.80s).

Gonzalo Casino (2003, p. 22) obvia el tema de la reforma sanitaria, y sitúa los comienzos del periodismo sanitario directamente en los años ochenta, unido en concreto a dos acontecimientos importantes:

"A principios de la década de los ochenta del siglo XX, el periodismo de salud, en sus vertientes biomédica y sociosanitaria, todavía no tenía entidad ni espacio propio en la prensa de información general española. Tampoco había, en general, periodistas especializados en temas de salud, como los podía haber, por ejemplo en cultura o deportes. Las noticias médicas no solo eran menos habituales que ahora sino, sobre todo, menos rigurosas y completas.

Pero dos nuevos frentes noticiosos que se abrieron a principios de los ochenta empezaron a exigir de forma continuada información técnica especializada: el Síndrome Tóxico por aceite de colza desnaturalizado (1981) y el Síndrome de Inmunodeficiencia Adquirida SIDA (1983) (...) Desde entonces, la

${ }^{5}$ Informe técnico que planteaba diferentes alternativas de reforma sanitaria en 1991, pero que finalmente fue desechado por los Grupos Parlamentarios 
información médica ha venido ocupando un espacio informativo creciente" (Casino. 2003, p.22)

Coral Larrosa (2003, p.43) recoge la misma idea que, a su vez, defienden Elena Barrena (2003, p. 73) e Idoia Camacho (2010, p.141):

"Hoy todos los medios de comunicación españoles tienen espacios específicos para la salud y la ciencia. Pero no desde hace mucho tiempo. La aparición del Síndrome Tóxico y el SIDA en los ochenta marcan un antes y un después (...) A partir de estos dos hechos, se crean espacios en las páginas, los guiones y las escaletas para la información sanitaria y de salud y se empieza a disponer de profesionales especializados"

La población comienza a utilizar los medios de comunicación como fuente para obtener información sobre temas concretos de salud y de costumbres saludables, contando con la función de divulgación médica del periodismo: se convierten en mediadores entre los médicos y el resto de la sociedad

"En conjunto, puede decirse que los periódicos han sido a la vez motor de la educación sanitaria de la población y reflejo de la misma. El creciente espacio informativo sobre salud en los diarios españoles es indicativo tanto del interés de la sociedad por estos temas como del creciente nivel de educación sanitaria de periodistas y lectores" (Casino, ídem, p.22)

Los periodistas científicos españoles, se quejaban de que, aún en los años 90 era excepcional ver una noticia científica en la prensa diaria (De Semir, 1990, p.175). Hubo casos inusuales, como el de la publicación de la revista mensual "Muy Interesante" $(1989)^{6}$

\section{SITUACIÓN EN LOS AÑOS 90}

Entre 1995 y 2000 nos encontramos con una situación diferente. El volumen de noticias científicas y médicas que aparecen en los medios de comunicación, aumenta de forma considerable. No obstante, "Pese a este incremento en el número de noticias científicas y médicas, el número de periodistas especializados en los grandes rotativos no ha variado, lo que representa una dificultad para mantener el nivel adecuado de calidad, rigor y profundidad en el tratamiento de la información" (Márquez 2010, p. 165).

A partir de 1995, las noticias sobre salud y medicina han ido ocupando cada vez más espacio en los medios de información general. El periodismo sanitario ejerce su influencia principalmente en las medidas preventivas que pueden adoptar los ciudadanos: actuar antes de que se produzcan las enfermedades y seguir hábitos

\footnotetext{
${ }^{6}$ Según la OJD, en 1991 tenía 1.816.000 lectores
} 
saludables. Por otro lado, la actividad periodística no es solo información, sino que es una actividad comprometida con la sociedad: la educación. En este caso, en salud.

Coincidimos con Antonio Alarcó (1999, p. 68) en que la divulgación biomédica se había convertido en una estrella en los medios de comunicación

"Ha relevado a las informaciones sobre física nuclear y la astronáutica, protagonistas indiscutibles en los años sesenta y setenta. Una de las explicaciones es el deseo de lograr una mejora en la calidad de vida por los ciudadanos, que va en consonancia con estar informado de los asuntos más candentes de la sanidad: el cáncer, las cardiopatías, enfermedades cerebrales o el SIDA entre otros (...) Hoy no parece plantear duda el mayor interés suscitado por una noticia referida al descubrimiento del remedio de enfermedades graves, que al descubrimiento de agua en la luna o una nueva misión espacial"

Elena Blanco y Francisco Javier Paniagua (2007, p.6) inciden en su importancia:

"Sin duda se trata de informar con rigor y seriedad, sin crear nunca falsas expectativas. Pero también, por qué no, se trata de educar en salud, con mensajes que también formen y sean de utilidad para la población, elaborados por profesionales responsables que eviten el sensacionalismo (...) En el periodismo de salud se maneja información compleja y delicada que puede tener un efecto contrario al que se pretende"

\section{EL PAPEL DE LAS ORGANIZACIONES : LA OMS}

La Organización Mundial de la Salud (OMS), es el organismo de la Organización de las Naciones Unidas (ONU) especializado en gestionar políticas de prevención, promoción e intervención en salud a nivel mundial. ${ }^{7}$

En 1995 comienza a publicar la OMS los Informes anuales sobre la Salud en el Mundo. A partir de 2005 cada año publica las Estadísticas Sanitarias Mundiales a través del Observatorio Mundial de la Salud, la puerta de acceso de la OMS a las estadísticas mundiales relacionadas con la salud

La Constitución de la Organización Mundial de la Salud de $1948^{8}$ define la salud como:

"Un estado de completo bienestar físico, mental y social, y no solamente la ausencia de afecciones o enfermedades"

${ }^{7}$ www.who.int Consultado: 5-12-2013

${ }^{8}$ La definición no se ha modificado desde entonces 
Desde esta organización, se viene pidiendo a los profesionales de la salud y a las administraciones públicas que faciliten el acceso de la sociedad a la información sanitaria. En su Asamblea del año 2004 se decidió crear una Comisión sobre Determinantes Sociales de la Salud ${ }^{9}$.

"Los determinantes sociales de la salud son las circunstancias en que las personas nacen, crecen, viven, trabajan y envejecen, incluido el sistema de salud. Esas circunstancias son el resultado de la distribución del dinero, el poder y los recursos a nivel mundial, nacional y local que depende a su vez de las políticas adoptadas. (...) explican la mayor parte de las inequidades sanitarias, esto es, de las diferencias injustas y evitables observadas en y entre los países en lo que respecta a la situación sanitaria"10

Para reducir la inequidad sanitaria entre países y dentro de los países, era necesario ir más allá de las causas inmediatas de la enfermedad. Así pues, la Comisión se interesaba por las "causas de las causas", los factores sociales que determinan la vida, el trabajo y el envejecimiento de la población:

"La mayor parte de los problemas de salud se pueden atribuir a las condiciones socio económicas de las personas. Sin embargo, en las políticas de salud han predominado las soluciones centradas en el tratamiento de las enfermedades, sin incorporar adecuadamente intervenciones sobre las causas de las causas, tales como, por ejemplo, las acciones sobre el entorno social. En consecuencia, los problemas sanitarios se han mantenido, las inequidades en salud y atención sanitaria han aumentado, y los resultados obtenidos con intervenciones en salud centradas en lo curativo han sido insuficientes y no permitirán alcanzar las metas de salud de los Objetivos para el Milenio."11

Cuando la OMS quiere ahondar en las "causas de las causas", está entrando es los determinantes sociales, en los aspectos socioculturales de los pueblos. No es tan sencillo hablar de salud, ya que depende de muchas variables entre las que está la atención sanitaria. Pero no es la única y aquí entra también la prevención como una cuestión más social que sanitaria.

En el Proyecto del Duodécimo Programa General de Trabajo de la OMS ${ }^{12}$ (mayo de 2012), encabezado por la última parte de la definición que la

${ }^{9}$ La Comisión se constituyó en Santiago de Chile en marzo de 2005

${ }^{10}$ www.who.int/social_determinants/es/CConsulta: 12-11-2013

${ }^{11} \mathrm{http} / /$ www.who.int/social_determinants/strategy/QandAs/es/index.html . Página web de la Comisión de Determinantes Sociales de la OMS en español. Consulta: 12 -112013

12 Proyecto para examen por los Comités Regionales para el período 2014-2019, presentado en mayo de 2012 a la $65^{\mathrm{a}}$ Asamblea Mundial de la Salud y al Consejo Ejecutivo en su $131^{\mathrm{a}}$ reunión. En 
organización hace de la salud: "No solo la ausencia de enfermedad" aparecen como principios fundamentales:

- La extensión a todos los pueblos de los beneficios de los conocimientos médicos, psicológicos y afines es esencial para alcanzar el más alto grado de salud

- Una opinión pública bien informada y una cooperación activa por parte del público son de importancia capital para el mejoramiento de la salud del pueblo

- Los gobiernos tienen responsabilidad en la salud de sus pueblos, la cual solo puede ser cumplida mediante la adopción de medidas sanitarias y sociales adecuadas

En lo que se refiere a la Asamblea Mundial de la Salud, el Proyecto de Programa General de Trabajo se resume en el siguiente gráfico, en cuya base aparecen los siguientes enunciados :

- Comunicación estratégica

- Gestión de los conocimientos

Imagen $n^{0}$ 5. Organigrama de la OMS

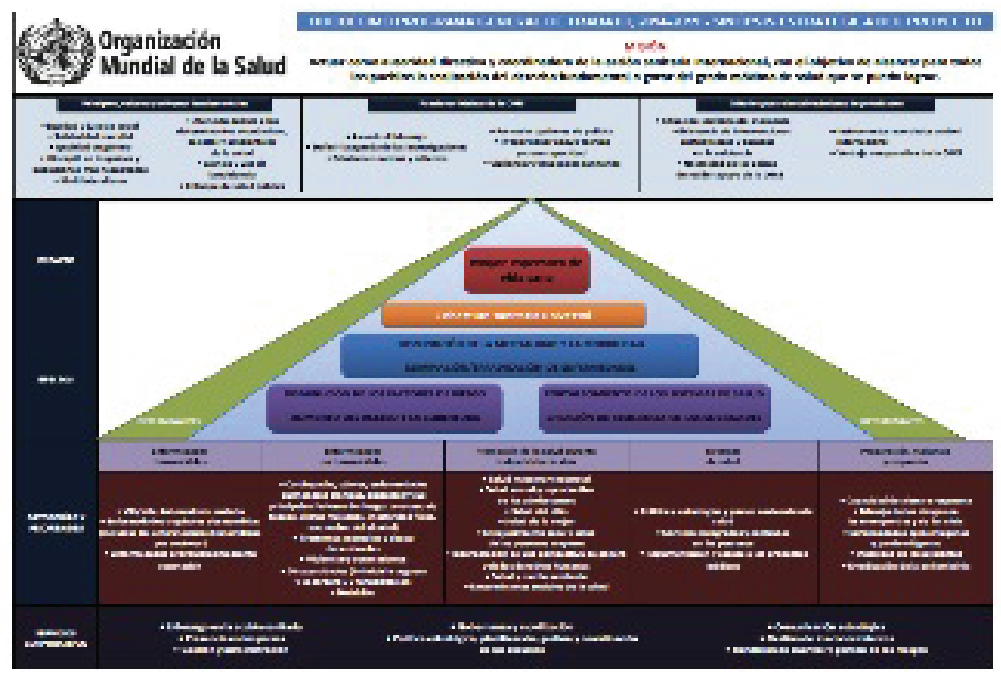

http://www.who.int/about/resources_planning/gpw_for_reg_commitees_2012_es.pdf Consulta 12-10-2013 
El último Informe General, el correspondiente al año 2012, examina el impacto de las investigaciones sanitarias:

"Durante los últimos años se han hecho esfuerzos internacionales continuos para alentar las iniciativas de investigación y de aplicación práctica de conocimientos en el campo de la salud pública. El Informe sobre la salud en el mundo 2012 destacará también lo importante que es la realización de investigaciones sanitarias y la aplicación de sus resultados para ayudar a fomentar comportamientos saludables" (OMS 2012)

Además de ofrecerlo a sus principales destinatarios (países miembros, organizaciones internacionales, organismos donantes), el informe va "destinado a una audiencia más amplia" en la que se encuentran las Universidades, los hospitales con actividades docentes, los periodistas y el público en general. La OMS es consciente de que el periodismo de salud interesa cada vez más a los ciudadanos y que es importante aprovechar esta demanda informativa para contribuir a su formación y promover los "comportamientos saludables"

\section{Investigación en Comunicación y Salud en España}

El Informe Quiral: El Observatorio de la Comunicación Científica y la Fundación Vilá Casas ${ }^{13}$

Con el objetivo de analizar en profundidad el tratamiento dado por los medios de comunicación a los temas de contenido biomédico y de salud, en 1996 se puso en marcha una iniciativa denominada "Proyecto Quiral", que centraba su principal actividad en la monitorización sistemática de la prensa española. Este estudio se viene presentando desde entonces con una periodicidad anual bajo el nombre de "Informe Quiral" elaborado conjuntamente por el Observatorio de la Comunicación Científica de la Universitat Pompeu Fabra (Barcelona) y la Fundación Vilá Casas bajo la dirección de Gemma Revuelta y Vladimir De Semir. En su Presentación de 2009, especifican:

"Es el resultado del estudio de las noticias sobre medicina y sanidad publicadas en la prensa española a lo largo de un año. Para ello se recogen sistemáticamente los contenidos de los cinco diarios de mayor difusión en España que son, según la OJD: El País, El Mundo, ABC, La Vanguardia y El Periódico. ${ }^{14}$ El objetivo de este análisis es la reflexión sobre el papel de los medios de comunicación en la transmisión de la información sobre salud a la sociedad en general. (...) El estudio centra su atención en diferentes aspectos:

${ }^{13} \mathrm{http}: / /$ www.fundaciovilacasas.com/es/salud-iinforme-quiral/ Consultado: 6-10-2013. Este enlace contiene informes desde 2000 hasta 2013 inclusive

www.upf.edu/pcstacademy/_docs/Quiral10.pdf. Este enlace incluye los informes desde 1997 hasta 2006 . Consultado: $\overline{6}-10-2013$

${ }^{14}$ En su primer informe, 1996, también se incluyeron los periódicos Avui y Eco 
qué temas han sido noticia a lo largo del año, cómo se ha presentado la información, cuáles han sido los puntos de debate, quiénes los protagonistas implicados, cómo ha reaccionado la opinión pública y qué consecuencias ha tenido la presencia de la noticia en los medios sobre la evolución del propio acontecimiento"

Como prueba del interés que despiertan los temas de salud entre el gran público, interés que recogen los periódicos, el "Informe" hace un resumen en su “Análisis de 10 años de información sanitaria: 1997-2006" (2007, p.13):

"Entre el 1 de enero de 1997 y el 31 de diciembre de 2006 se publicaron, en los cinco diarios de la muestra, 88.555 textos $^{15}$ referidos a salud y medicina. Durante la primera mitad del decenio, el número de textos fue incrementándose año tras año. Durante la segunda mitad, la cifra se estabilizó. Aunque siempre manteniéndose en un nivel dos veces mayor que en el primer año. (...) al finalizar el año 2006, tres de los cinco diarios disponían de secciones diarias o semanales dedicadas específicamente a la salud o a la ciencia (El País, El Mundo y ABC) y dos disponían de Suplementos sobre salud (El Mundo y El País)"

A pesar de este volumen de textos y entre las conclusiones que veremos a continuación, en la $\mathrm{n}^{\mathrm{o}} 6$ se hace notar que la información se concentra en un grupo muy reducido de temas, los que son más mediáticos, la actualidad del momento: la agenda social. Junto a estos datos, la primera de sus Conclusiones generales supone un recorrido por la situación del tema salud en esa década (Informe Quiral 1997-2006, p. 122)

"Entre 1997 y 1999 se produjo un aumento muy acelerado del interés por los temas relacionados con la salud y la medicina en los diarios estudiados. A partir de 2000 dicho interés no crece de un año a otro, pero se mantiene constantemente elevado (...) Se descarta que este aumento en el espacio dedicado a la salud durante el decenio, sea debido a un aumento del espacio general de los diarios. En realidad la tendencia de éstos, a lo largo de los años estudiados, ha sido justo la contraria: se ha reducido el número total de páginas. (...) Se observa, sin embargo, un paralelismo claro entre la curva de evolución del interés por la salud en la prensa y la penetración en España de las TIC (...) Se concluye que el aumento del interés de la prensa por los temas de salud y medicina entre 1997 y 2006 puede haber sido debido, en parte, a la difusión de las TIC en nuestro país"

Respecto a las Conclusiones Generales que extraen a lo largo del estudio de la década, resaltamos lo siguiente:

${ }^{15}$ No se contabilizan aquí los "breves" 
1) El aumento del interés por la salud en la prensa en el decenio 1997-2006, es paralelo a la penetración y difusión de las TIC en España

2) En los últimos años se incluyen en los diarios más secciones especializadas en ciencia y/o salud (más en formato de sección que en el de suplemento). La mayor parte de la información sobre salud y medicina sigue siendo tratada en el conjunto del diario en la misma forma en que lo ha hecho durante décadas: en secciones generalistas. En los últimos años se empieza a observar una apuesta por la creación de secciones especializadas al mismo nivel que las del resto del diario.

3) La salud se trata fundamentalmente desde la perspectiva de un periodismo informativo, sin la contextualización o la reflexión experta que facilitan los géneros de opinión e interpretación, más presentes en otros temas de la actualidad

4) A lectores, articulistas y editores les interesan temas distintos. Los primeros son los que hablan más del día a día de la asistencia, los segundos, de las biotecnologías y los últimos de las epidemias. El estudio de las cartas al director, los editoriales y los artículos de opinión, muestra la existencia de tres perfiles de interés distinto en cuanto a los temas que se tratan con mayor frecuencia

5) La sección de cartas al director puede ser utilizada de forma estratégica por los lectores para llegar a los responsables políticos del sistema sanitario ${ }^{16}$

6) Aunque aparentemente la prensa haya tratado temas de salud muy diversos a lo largo de este decenio, la información en realidad se concentra en unas pocas cuestiones más mediáticas que son las que marcan a su vez la agenda social.

7) Las decisiones sobre qué es noticiable o no en materia de salud, están regidas por los mismos criterios que en el resto de ámbitos de la actualidad: los valores noticia, los procesos de producción y las relaciones entre los agentes de comunicación.

8) La observación de la existencia de dos patrones distintos de cobertura periodística de los temas médicos y sanitarios -Patrón Agudo y Patrón Crónico- ${ }^{17}$ plantea cuestiones sobre su distinta influencia en el público

9) La existencia de tres modalidades de relación entre la Administración y los medios -Presión, Alianza y Utilitarismo- ${ }^{18}$ sugiere distintos tipos de influencias en la evolución de los acontecimientos

${ }^{16}$ Lo que en término anglosajón se denomina media advocacy, (utilización estratégica de los medios)

${ }^{17}$ Terminología utilizada en el Informe Quiral desde su primera investigación. El Patrón Agudo se corresponde con noticias cortas y poco especializadas y el Patrón Crónico con un tratamiento periodístico más especializado y elaborado

${ }^{18}$ Denuncia periodística, alianza para promocionar comportamientos saludables, publicación -sin implicarse- de la información procedente de la Administración 
10) Las fuentes de información se concentran en unos sectores y en unas personas concretas. Los periodistas utilizan a sus fuentes para dar credibilidad a su trabajo y éstas utilizan a los medios para dar visibilidad a la información que quieren hacer pública.

11) Internet ha cambiado muchos aspectos de la relación del ciudadano con la información y la gestión de su salud, incluyendo su participación en foros de pacientes y su interacción con la propia prensa

A partir de 2009, cambia su planteamiento cara el período 2010-20. Su investigación se modifica, según sus propias palabras, "debido a la gran transformación experimentada durante los últimos años en los hábitos de consumo informativo de la ciudadanía" (Informe Quiral 2011, p. 5) e incorpora las siguientes novedades:

a) El informe deja de estudiar únicamente prensa, para adoptar un análisis multimedia (prensa, radio, televisión, medios electrónicos y redes sociales)

b) No solo se analiza la información de medios españoles, sino también internacionales

c) En lugar de seguir todos los contenidos de salud, el informe tiene actualmente un enfoque monográfico

En 2010, se analizó la enfermedad de Alzheimer. En 2011 han presentado un análisis del cáncer. Su elección se basa en el hecho de que sean patologías de gran impacto social, cuya prevalencia está en aumento y su presencia en los medios de comunicación muerte a escala mundial según los datos aportados por la $\mathrm{OMS}^{19}$.

${ }^{19} \mathrm{Al}$ cáncer se le atribuyen 7,6 millones de defunciones (aproximadamente el 13\% del total) ocurridas en todo el mundo en 2008. Se prevé que el número de defunciones por cáncer siga aumentando en todo el mundo y supere los 13,1 millones en 2030 
Imagen $\mathbf{n}^{\mathbf{0}}$ 6. Temas que constituyen la actualidad biomédica y de salud entre 1997 y 2006 (frecuencia = a número de textos periodísticos centrados en el tema en cuestión). Revuelta y Minelli (2008, p. 59)

\begin{tabular}{lc}
\multicolumn{1}{c}{$\begin{array}{c}\text { Temas } \\
\text { POLÍTICA SANITARIA: listas de espera, negligencias, hospitales, gasto farma- }\end{array}$} & $\begin{array}{c}\text { Frecuencia } \\
\left(n^{\circ} \text { textos }\right)\end{array}$ \\
$\begin{array}{l}\text { céutico, reforma sanitaria, «medicamentazo», gestión y política sanitaria, colec- } \\
\text { tivo sanitario, MIR }\end{array}$ & 13.797 \\
\hline $\begin{array}{l}\text { LAS CINCO ENFERMEDADES MÁS «MEDIÁTICAS»: sida, cáncer, diabe- } \\
\text { tes, anorexia y Alzheimer }\end{array}$ & 7.171 \\
\hline $\begin{array}{l}\text { BIOTECNOLOGÍA: células madre, clonación, genoma, genética, embriones, te- } \\
\text { rapia génica, transgénicos }\end{array}$ & 5.249 \\
\hline EPIDEMIAS: «vacas locas», SARS, gripe aviar, legionela & 5.094 \\
\hline CUIDADOS DE LA SALUD: dieta, nutrición, alimentación, calidad de vida & 4.242 \\
\hline TABACO & 3.405 \\
\hline $\begin{array}{l}\text { FÁRMACOS E INDUSTRIA FARMACÉUTICA: fármacos, industria farma- } \\
\text { céutica }\end{array}$ & 3.376 \\
\hline REPRODUCCIÓN Y SEXUALIDAD & 2.906 \\
\hline DROGAS & 2.454 \\
\hline EUTANASIA & 1.292 \\
\hline TOTAL & $\mathbf{4 8 . 9 8 6}$ \\
\hline
\end{tabular}

En conjunto, el informe nos permite conocer los contenidos que más interesan a los medios de comunicación. Vemos bastante coincidencia entre las agencias temáticas de los medios de comunicación analizados. Y ellos mismos advierten que los temas resultantes de las cartas al director que envía el público, no coinciden con los temas que exponen los medios

\section{El Observatorio de Comunicación y Salud: Instituto de Comunicación InCom de la Universidad Autónoma de Barcelona \\ El Observatorio Comunicación y Salud (OCS) ${ }^{\mathbf{2 0}}$ se creó en 2005.Depende del} Instituto de Comunicación (InCom) de la Universidad Autónoma de Barcelona. Tiene como finalidad el estudio de la comunicación en relación con la salud respecto a tres ejes:

- La comunicación entre sujetos

- La comunicación entre instituciones y sujetos

- El análisis de la información sociosanitaria que transmiten los medios de comunicación de masas

Junto a la investigación, el OCS asume en la Presentación de su página web los objetivos de la divulgación, la formación y la organización de eventos en el ámbito de la comunicación y la salud. Para conseguir sus objetivos, plantea sus actividades de manera interdisciplinar y transdisciplinar. Pretende ser un lugar de encuentro de investigadores de la comunicación, periodistas y personal e instituciones sanitarias. Entre los múltiples recursos que encontramos en su web, aparece su historial de investigaciones relacionadas con la salud en los medios, historial que comienza el mismo año de su puesta en funcionamiento. Entre sus

\footnotetext{
${ }^{20}$ www.portalcomunicacion.com/ocs/ Consulta: 12 -11-2013
} 
últimos recursos actualizados, encontramos diversos artículos en torno a estos temas: "Comunicar sobre salud", "La salud en los medios" y "Promoción de la salud. Campañas".

Sus líneas de investigación, siguiendo su propia presentación en la web, son:

- La influencia del marketing y de la publicidad en conductas y hábitos de vida

- La comunicación entre el personal sanitario o las instituciones médicas y los pacientes

- La comunicación entre pacientes

- La comunicación entre los profesionales de la sanidad

- Las relaciones entre las instituciones públicas y privadas y los medios de comunicación de masas

- El análisis de cómo se trata la salud en los medios de comunicación de masas

- El marketing social para la promoción de la salud

- El periodismo especializado en temas de salud

- Los roles de internet y el web en el entorno de la salud

\section{El Instituto Internacional de Comunicación y Salud_(INICyS)}

Según figura en la presentación de su página web, el Instituto Internacional de Comunicación y Salud (INICyS) es una organización sin ánimo de lucro que nació "como iniciativa de un colectivo de académicos y profesionales que venían trabajando de forma colaborativa desde hace casi una década ${ }^{21}$ en el campo de la comunicación y la salud"22 (...) En línea con su objetivo de promover el desarrollo de conocimientos, herramientas y acciones en materia de comunicación y salud, INICyS desarrolla en colaboración con otras instituciones y empresas, una amplia variedad de actividades:

- Publicaciones científicas sobre comunicación y salud en las que se abordan los últimos modelos y tendencias en este campo (...) destaca la Revista de Comunicación y Salud $^{23}$, publicación científica de ámbito iberoamericano y los libros de Comunicación y salud editados en colaboración con la UCM

- Consultoría y asesoramiento (...) para el desarrollo de estrategias y programas de comunicación y salud

${ }^{21}$ No se menciona fecha de fundación

${ }^{22}$ www.inicys.com/ Consulta: 15-11-2013

${ }^{23}$ Sale por primera vez en enero de 2011. Se difunde electrónicamente en formato de libre acceso. En su presentación, se dice que tiene como objetivo "contribuir al desarrollo de la comunicación y la salud en Iberoamérica, ofreciendo un espacio de intercambio científico a los investigadores y una rigurosa guía de buenas prácticas a los profesionales” 
- Formación de grado y posgrado ${ }^{24}$ (...) en colaboración con la UCM, el organismo autónomo Madrid Salud, la Fundación Abbot y la Fundación de Educación para la Salud (FUNDADEPS) entre otras"

Sus antecedentes los encontramos en 2005, en los cursos especializados “Comunicación y Salud", de cuya organización se encargaban tres organismos:

- La Facultad de CC de la Información de la Universidad Complutense de Madrid (Departamento de Comunicación Audiovisual y Publicidad II)

- "Madrid Salud" organismo autónomo del Ayuntamiento de Madrid

- La Fundación privada Abbot

Con esta colaboración, se desarrollaron varios cursos universitarios desde 2005 a la actualidad. Aitor Ugarte, uno de los responsables del curso, sitúa su génesis en el organismo "Madrid Salud", que agrupa todas las competencias en materia de salud pública y adicciones del Ayuntamiento de Madrid. Dentro de "Madrid Salud" se creó el Grupo de Trabajo en Comunicación y Salud que comenzó una labor de investigación diversificada a partir de 2007: (Ugarte 2008, pp. 46s)

Los primeros intereses del Grupo de Trabajo giraban alrededor de la comunicación de riesgo y de crisis, de la convalidación de las teorías de la agenda setting y del framing siempre aplicados a la ciudad de Madrid y en asuntos relacionados con la salud.

Otra línea de investigación inició la colaboración con el Departamento de Comunicación Audiovisual y Publicidad II de la Facultad de Ciencias de la Información de la Universidad Complutense de Madrid. Se trataba de una investigación de comunicación de masas que, siguiendo la línea del "Informe Quiral", analizaba los contenidos de salud de:

- Periódicos gratuitos de Madrid (Qué, 20 Minutos, Metro, ADN)

- Las páginas de la Sección de "Madrid" de 4 diarios nacionales ( $E l$ País, El Mundo, ABC, La Razón)

El resultado de esa colaboración se amplió con la de la Fundación Abbot, que en 2005 había organizado el I Curso sobre Comunicación y Salud junto con la Fundación General de la Universidad Complutense y el Área de Gobierno de Seguridad y Servicios a la Comunidad del Ayuntamiento de Madrid ${ }^{25}$.

La Fundación Abbot es una institución sin ánimo de lucro, cuyos objetivos se especifican en su página web:

"Contribuir a la mejora de la salud de la población a través de la puesta en marcha de distintas iniciativas encaminadas a atender las necesidades del sistema sanitario español, de sus profesionales y de la sociedad en general." Desde 2005 a 2012 se han desarrollado un total de ocho cursos compartiendo organización, y se han publicado cuatro libros en relación con sus contenidos:

\footnotetext{
${ }^{24}$ Magister de Comunicación y Salud (comienzo en 2013)

${ }^{25}$ Noticias de la Fundación. Consulta 10-10-2013
} 
- Comunicación Social y Salud. Una introducción. (2005) VVAA. Coordinación: Ubaldo Cuesta Aitor Ugarte y Tania Menéndez. Madrid. Ed. Complutense

- Comunicación y Salud. Avances en modelos y estrategias de intervención (2008) Ubaldo Cuesta, Aitor Ugarte y Tania Menéndez. Madrid. Ed. Complutense

- Prensa escrita y salud en Madrid (2009) Aitor Ugarte, Tania Menéndez y Ubaldo Cuesta. Madrid. Ed. Complutense

- Comunicación y Salud. Nuevos escenarios y tendencias (2011).VVAA. Coordinadores: Ubaldo Cuesta, Tania Menéndez y Aitor Ugarte. Madrid. Ed. Complutense

\section{El Seminario de Comunicación y Salud de la Universidad de Navarra}

Desde 2004 hasta la actualidad, la Facultad de Comunicación de la Universidad de Navarra (inicialmente a través del Departamento de Comunicación Pública) y el Instituto Novartis de Comunicación en Biomedicina (INCB) ${ }^{26}$ han venido organizando cada año un Seminario sobre Comunicación y Salud con una duración que varía entre dos y tres meses

En 2013 se ha desarrollado la décima edición del Seminario bajo el título de "Comunicación y Salud: hacia la convergencia de una extraña pareja". A través de este Seminario, Novartis resalta la importancia del inseparable binomio comunicación-salud. Del mismo modo, pone de relevancia cómo la información sanitaria influye en el cuidado del ciudadano, que cada vez demanda más información de calidad sobre salud a los medios de comunicación.

Según se expone en su página $\mathrm{web}^{27}$, el Instituto Novartis de Comunicación en Biomedicina fue creado en 2001 con la finalidad de aglutinar actividades alrededor de la comunicación en salud y biomedicina.

El INCB resalta su compromiso con la formación. Está vinculado a organismos e instituciones estrechamente relacionados con medios de comunicación, universidades

así como aquellos colectivos interesados en la formación y difusión de contenidos y proyectos en salud y biomedicina. Su propósito es promover, articular y desarrollar actividades de complemento a la docencia, investigación, formación y documentación en el ámbito de la Comunicación en Salud y Biomedicina. Asimismo aportar, compartir y divulgar el conocimiento, a través de medios actuales y tecnologías emergentes.

Consultado: 12-11-2013 http://www.novartis.es/sala-de-prensa/fee59060-c34f-4851-89cad6dee7052e14

${ }^{27}$ www.novartis.es Consulta 7-12-13 


\section{Grupos de Trabajo sobre Comunicación y Salud de la Sociedad Española de Medicina Familiar y Comunitaria (SEMFyC)}

La Sociedad Española de Medicina Familiar se funda a finales de los años 70. La SEMFyC, según se determina en su página web, ${ }^{28}$ es una asociación científica médica privada y sin ánimo de lucro, centrada -como su nombre indica- en la medicina de familia y comunitaria. Su estructura es federada y su organización se articula en secciones, grupos de trabajo y programas específicos.

\section{Los Grupos de Trabajo}

En 1989 se funda el primer Grupo de Trabajo sobre Comunicación y Salud perteneciente a esta Sociedad

El Grupo de Trabajo se transforma en Programa de Comunicación y Salud ${ }^{29}$ en el año 2007 como uno de los tres pilares esenciales de la Medicina de Familia "con el propósito de ayudar a desarrollar las capacidades y herramientas necesarias que sirvan a los profesionales sanitarios para humanizar la asistencia sanitaria y mejorar la comunicación asistencial. Se perfila como un instrumento para que la semFYC alcance influencia técnica y social en las instituciones sanitarias y formativas, en sociedades científicas y ante los ciudadanos, impulsando su presencia como agente formativo en el campo de la relación asistencial y comunicación clínica."

Existen un total de 10 Grupos de Trabajo Autonómicos: Baleares, Canarias, Cantabria, Castilla-León, Catalunya, Galicia, Euskadi, La Rioja, Madrid y Murcia

Los Grupos de Trabajo CyS, actuales Programas de CyS, llevan años desarrollando actividades de autoformación en Docencia en Comunicación así como investigaciones

"La transformación del grupo CyS en programa de la semFYC, supone un avance (...) Los recursos de información, los equipos de trabajo del propio grupo CyS y las infraestructuras de semFYC se integran directamente en un proceso de difusión de contenidos, noticias, actividades, contactos, enseñanza/aprendizaje y materiales educativos sobre comunicación asistencial que posibilitan el desarrollo curricular de la misma entre los interesados que contacten con el Grupo o la Sociedad Científica directamente, tanto a nivel nacional como Autonómico e Internacional, interesados en la Comunicación asistencial” (P. web, ídem, “Quienes somos")

${ }^{28}$ www.semfyc.es Consulta 15-11-2013

${ }^{29} \mathrm{http}$ :/comunicacionysalud.es/ Consulta 15-11-2013 


\section{Investigadores diversos del binomio "Comunicación y Salud" Investigaciones en ámbitos más localizados}

Hasta ahora hemos visto investigaciones de ámbito estatal e incluso internacional. Queremos hacer alusión concreta a investigaciones más localizadas geográficamente. Nos ceñimos a la prensa y no encontramos demasiado contenido:

- María Reyes León Vergara, Manuel Herrera Usagre, Rosario M. Delgado Ramos $^{30}$

Esta investigación la lleva a cabo el equipo de Comunicación de la Agencia de Calidad Sanitaria de Andalucía, fundación pública encargada de la acreditación de calidad de los diferentes elementos del sistema sanitario:

"La Agencia de Calidad Sanitaria nace en 2002 en el Marco del Modelo Andaluz de Calidad impulsado por la Consejería de Salud de la Junta de Andalucía (...) y responde al compromiso social de ofrecer unos servicios de calidad que satisfagan las necesidades y expectativas de los ciudadanos". (pg. 7)

Realizada en 2010, se publicó en 2012 bajo el título Calidad sanitaria y mass media. El impacto mediático de la localización de notas de prensa sobre Acreditación Sanitaria en Andalucía bajo la premisa de que el empleo de estrategias dirigidas a conseguir una mayor eficacia de la comunicación, debe ser un factor fundamental en una organización del ámbito de la salud.

Se precisa una valoración sobre el cambio de estrategia llevado a cabo en 2010 por este equipo. Para ello se hace:

- El análisis de las notas de prensa emitidas y de las notas publicadas sobre acreditación de calidad

- El recuento de centros y profesionales sanitarios acreditados en salud en el conjunto de la Comunidad Autónoma de Andalucía

La conclusión a la que se ha llegado, es que ha habido un incremento significativo de noticias publicadas sobre acreditación de calidad en salud en el conjunto de la Comunidad Autónoma de Andalucía. A su vez, este grupo de investigación hace una reflexión importante:

"Hasta hace relativamente poco, la comunicación en el ámbito de la salud estaba relegada a la gestión de las crisis sanitarias y a amortiguar las informaciones negativas que aparecían en los medios de comunicación. En estos momentos, sin embargo, la comunicación en este campo tiene una actitud proactiva, abarcando una dimensión nueva como son las acciones de promoción y prevención de la salud y la difusión de los logros científicos,

\footnotetext{
${ }^{30}$ www.revistadecomunicacionysalud.org/index.php/rcys/search/titles Consulta: 5$11-2013$
} 
técnicos y profesionales que pueden mejorar la salud de la ciudadanía en general o de diferentes colectivos"

- Laura Hernández García ${ }^{31}$

Publicó en 2011 los resultados de una investigación titulada Análisis de los contenidos sociosanitarios en prensa local, llevada cabo en la ciudad de Almería y cuyo objetivo era realizar un análisis descriptivo de los contenidos sociosanitarios en la prensa escrita local, profundizando en aspectos como:

- Espacio que se le dedica

- Quien las escribe

- Fuentes de información que utilizan

Utiliza un método de estudio descriptivo longitudinal para una muestra constituida por las ediciones diarias a lo largo de un mes de los periódicos Diario de Almería y La Voz de Almería.

Los resultados que expone no son muy alentadores y concluye manifestando la necesidad de que haya periodistas especializados en salud para transmitir de forma eficaz la información a la población:

- No llega al 7\% el espacio dedicado a los contenidos sociosanitarios

- Casi el $50 \%$ de las noticias fueron escritas por periodistas no especializados

- Los periodistas utilizaron fuentes de información solo en un $41 \%$ de los contenidos

- Los temas más frecuentes fueron los de violencia de género, IVE, aperturas y mejoras de centros sanitarios, ayuda a domicilio y formación soociosanitaria

- Carmen Costa Sánchez

Publicó en 2008 los resultados de una investigación sobre las noticias de salud en Galicia titulada Medicina y salud en la prensa. Las noticias de salud en los principales diarios de Galicia, basándose en: El Progreso, El Faro de Vigo, La Región y La Voz de Galicia ${ }^{32}$

Es un trabajo que tiene como objeto la investigación de la información sobre salud y medicina publicada a lo largo de una semana, entre julio de 2006 y junio de 2007, en los cuatro diarios mencionados, los de mayor difusión de Galicia según estimación OJD. La autora hace un análisis de lo que ocurre en las redacciones de las principales cabeceras generalistas de España, observa cómo van apareciendo secciones especializadas en salud y se incorporan periodistas también especializados en el tema y se pregunta qué ocurre en la

\footnotetext{
${ }^{31}$ Disponible en: http://www.aecs.es Consulta, 15-11-2013

${ }^{32} \mathrm{http}: / / w w w . u l l . e s /$ publicaciones/latina/_2008/03/Costa_Sanchez.html. Consultado: 19-11-2013
} 
prensa autonómica: ¿Cuál es el tratamiento informativo que recibe la información sobre salud y medicina en la prensa diaria (y más difundida) de Galicia?

Su estudio se hace en base a aspectos descriptivos, cuantitativos y de análisis de contenido, que le permitirán reflexionar sobre la cobertura informativa que reciben estas noticias con la intención de hacer un diagnóstico de la situación y que le lleva a la siguiente consideración:

"El presente trabajo nos ha permitido comprobar que la información de medicina y salud se encuentra en la actualidad en un estadio que podemos calificar de primario en la prensa diaria gallega o, lo que es lo mismo, que aún queda por delante un largo camino que recorrer"

La autora basa esta conclusión en un tratamiento informativo caracterizado por:

"1. La dispersión entre las secciones de Sociedad y las de criterio geográfico (Galicia, local...), que resta peso y visibilidad a dicha información en el conjunto del temario.

2. La elección de géneros predominantemente informativos, que libera al periodista de la función de documentarse e interpretar y ampliar los datos para sus lectores.

3. Cuantitativamente, la media de información publicada supera la página diaria, lo que indica un cierto interés por la cobertura informativa de esta área (...) Sin embargo, se trata de información muy parcelada(...) que perjudica las posibilidades de contextualización y profundización en la información.

4. El análisis de contenido refuerza la idea de que el tratamiento informativo llamativo y anecdótico se produce junto con (...) el seguimiento de los medios de actividades de representantes de la Administración pública, de actividades de debate y formación (...) o incluso de lo que marque el calendario internacional, en cuanto a enfermedades: Día Mundial de, etc.

5.Implica que los medios todavía funcionan por arrastre de una actualidad que marcan otros actores, en lugar de tomar la iniciativa de determinar lo que pueda ser interesante para sus lectores en materia de salud y medicina. Por supuesto, ello está muy vinculado a la presencia o ausencia de periodistas especializados en la Redacción, algo que, de momento, no es lo habitual en los diarios de Galicia.

6. (...) Se emplea una sola fuente de información en la mayoría de los textos analizados que es, habitualmente, el médico. Concebido como un sabio, su discurso se transmite en cita directa -evitándose así nuevamente la interpretación y el papel activo por parte del periodista- y se contrasta (o más bien se corrobora), mayoritariamente con el de otros médicos, esto es, otros expertos que se mueven en el mismo nivel de conocimiento. 
7. Se puede afirmar, aunque no de manera categórica ya que existe una dependencia directa con la actualidad, que determinadas especialidades médicas están de moda, destacando: la Oncología, la Cirugía plástica, la Nutrición, la Ginecología o la Psiquiatría.

8. La prensa se interesa por la Sanidad con un espíritu de denuncia (...) dan voz a las denuncias sociales (...) La explicación de este factor también ha de buscarse en (...) la dinámica de las bad news.

9. Los valor noticia relevancia y proyección, adecuados a priori a la importancia de los hallazgos médicos, son los menos habituales en la muestra analizada. Predominan novedad, actualidad, conflicto y proximidad geográfica. 10. No se prodigan los textos de divulgación informativa, probablemente a consecuencia de la dinámica de producción de la información que suma política y agenda del día (...)" (2008 pp. 19s)

Existe, por último, una tesis doctoral investigada por Elena Becerra Muñoz y presentada en 2006 en la Facultad de Comunicación de la Universidad de Málaga con el título Gestión de la comunicación en las instituciones sanitarias andaluzas que no hemos podido consultar directamente. Su contenido gira en torno a la imagen y la gestión de la sanidad en Andalucía y su reflejo en la prensa, según la sinopsis que se ofrece ${ }^{33}$ :

"La información relacionada con la salud genera una gran alarma social y sitúa al sector y a sus instituciones en situaciones de crisis que contribuyen a la formación de la imagen negativa del sector. Esta tesis doctoral expone la situación en la que se encuentra el sector sanitario, el desarrollo que ha sufrido en los últimos años y los nuevos retos y necesidades que se le plantea. A través de una amplia investigación que analiza la prensa escrita -como generador de imagen y opinión pública- se presenta el reflejo que realizan acerca de los principales hospitales de Andalucía".

Esta investigación ha sido consultada y utilizada por el equipo de Comunicación de la Agencia de Calidad Sanitaria de Andalucía formado por María Reyes León Vergara, Manuel Herrera Usagre, Rosario M. Delgado Ramos en su investigación realizada en 2010 y publicada en 2012 Calidad sanitaria y mass media. El impacto mediático de la localización de notas de prensa sobre Acreditación Sanitaria en Andalucía, figurando el nombre de la autora y los datos de la investigación en su bibliografía 


\section{Libros sobre "Comunicación y Salud"}

- Comunicación y Salud. Nuevos escenarios y tendencias (2011).VVAA. Coordinadores: Ubaldo Cuesta, Tania Menéndez y Aitor Ugarte ${ }^{34}$

Este libro, cuarta monografía resultado de los Cursos especializados "Comunicación y Salud" llevados a cabo entre "Madrid Salud", la UCM y la Fundación Abbot,, aborda cuestiones como la creciente relevancia de la Comunicación y Salud entendida de forma integral, ante la demanda de campañas de publicidad y marketing de salud más evaluadas, testadas y soportadas sobre bases psico-sociales científicas; el reconocimiento de los profesionales sanitarios en general, y los médicos en particular, de la importancia de las habilidades de comunicación interpersonal para ser eficaces en su trabajo; o las actividades de salud grupal y comunitarias que comienzan a dar cabida al cuidado de las relaciones y las interacciones con los diferentes públicos.

- Prensa escrita y salud en Madrid (2009): Aitor Ugarte, Tania Menéndez y Ubaldo Cuesta ${ }^{35}$

Este libro, tercera monografia resultado de los Cursos especializados "Comunicación y Salud" llevados a cabo entre "Madrid Salud", la UCM y la Fundación Abbot, introduce el estudio de los procesos informativos de la prensa escrita dentro del ámbito de los determinantes socio-culturales de la salud de una población, defendiendo la pertinencia de una acción comunicativa bien planificada y evaluada como una herramienta fundamental para mejorar la calidad de vida y el bienestar de una colectividad.

- La especialización en el periodismo. Formarse para informar. (2010) VVAA. Coordinadora: Idoia Camacho.

Es un manual centrado en la especialización del periodismo, en general, como su título indicdel mayor número posible de elementos de juicio que le permitan formarse una conciencia crítica sobre lo que acontece en el mundo. En esta dinámica, hay una parte importante dedicada al Periodismo de Salud.

- Noticias sobre salud y medicina: mucho más que información (2010) Idoia Camacho En La especialización en el periodismo. Formarse para informar. VVAA. Coordinadora: Idoia Camacho.

Aporcentual de temas de salud en los medios de comunicación, tanto generales como especializados:

\footnotetext{
${ }^{34} \mathrm{http}: / /$ www.fundacionabbott.es/publicaciones01.html). Consultado: 27-11-2013

${ }^{35} \mathrm{http}$ ://www.fundacionabbott.es/publicaciones01.html. Consulta: 20-11-2013
} 
"Los grandes diarios (y cada vez más otros medios impresos de menor entidad) han creado secciones y/o suplementos especializados en este tema; han aparecido nuevos programas de radio y televisión, así como nuevas publicaciones especializadas; comienzan a surgir agencias de comunicación especializadas en el sector sanitario, etc." (p. 142)

La autora hace un recorrido entre los distintos tipos de medios: prensa diaria y semanal, TV, radio, revistas especializadas e Internet. Su conclusión es: "a mayorla prevención "en subrayar la necesidad de actuar antes de que se produzcan las enfermedades (...) sentando las bases para que la sociedad no incorpore a su forma de vida hábitos que le pueden ser perjudiciales, o para que modifique aquellos que no son saludables" (p. 147)

- Comunicación y Salud: avances en modelos y estrategias de intervención. (2008). VVAA. Coordinadores: Ubaldo Cuesta, Aitor Ugarte y Tania Menéndez ${ }^{36}$

Esta segunda monografía resultado de los Cursos especializados "Comunicación y Salud" llevados a cabo entre "Madrid Salud", la UCM y la Fundación Abbot, ofrece avances significativos en este campo, presentando la perspectiva de especialistas del área en sus diferentes ámbitos de especialización, al mismo tiempo que ofrece una amplia descripción del "estado de la cuestión", cada día más relevante, de la Comunicación social y la Salud

- El Periodismo Científico en la prensa. Aspectos de biomedicina (2005) Antonio Alarcó Hernández y María Dolores Meneses Fernández.

Este libro está elaborado sobre el antecedente de la tesis doctoral de Antonio Alarcó presentada en 1999 en la Universidad de La Laguna con el mismo título: "El Periodismo Científico en prensa diaria Aspectos de biomedicina" ${ }^{37}$. En esta primera edición publicada junto a $\mathrm{M}^{\mathrm{a}}$ Dolores Meneses, los autores nos muestran la triple función que han de desarrollar los periodistas científicos: como divulgadores de la información científica y tecnológica, como intérpretes que precisen la significación de los descubrimientos e, incluso, como agentes del control social, para que las decisiones políticas no se tomen sin tener en cuenta los hechos científicos.

Con el principal objetivo de analizar la importancia del periodismo científico en España en los últimos 20 años, se aplica un método científico para medir el peso de las noticias de biomedicina en tres periódicos representativos; dos de tirada nacional: "El Mundo", "La Vanguardia" y uno local (Tenerife): "El Día".

Los datos obtenidos desvelan la gama variada de asuntos de salud tratados, lo que muestra la relevancia que tienen en la prensa diaria. También se analizan las actividades realizadas por instituciones y organizaciones. En su marco teórico

\footnotetext{
${ }^{36}$ http://www.fundacionabbott.es/publicaciones01.html. Consulta: 20-11-2013

${ }^{37} \mathrm{ftp}: / /$ tesis.bbtk.ull.es/ccssyhum/cs80.pdf . Consulta: 28-10-2013
} 
realiza un extenso informe sobre la necesidad de colaboración entre la comunidad científico-médica y los profesionales de la información

- Evolución de la información sanitaria en la prensa escrita durante la Transición española (1977-2002) (2005). Enrique Jurado Salván.

Esta obra está publicada a partir de la tesis doctoral que, con el mismo título, defendió el autor en la UCM $2004^{38}$. En ella, se pretende analizar la evolución de la información sanitaria española en la prensa escrita durante la Transición. Este largo periodo ha sido rico en acontecimientos sanitarios, muchos de ellos caracterizados por situaciones de crisis como el síndrome tóxico, el sida o las vacas locas. La conclusión que se extrae es que la información sanitaria en la prensa española no ha logrado situarse en un gran protagonismo periodístico. Únicamente, cuando se producen "sucesos sanitarios" o situaciones de crisis, los medios abordan la información en portada o mediante editoriales. En el resto de las ocasiones, la información sanitaria desaparece u ocupa lugares secundarios dentro de las secciones de Sociedad. La obra ha abordado dos estudios de campo sobre los cuatro periodos informativos de la Transición Sanitaria, analizando a los dos periódicos diarios de máxima difusión e influencia en este periodo: ABC y El País. La otra investigación ha comparado situaciones sanitarias de crisis en años tan dispares como 1981 y 2001, para analizar el tratamiento informativo sobre el síndrome tóxico y las vacas locas.

- Comunicación social y salud: una introducción (2005). VVAA. Coordinadores: Ubaldo Cuesta, Aitor Ugarte y Tania Menéndez ${ }^{39}$

Esta monografía recopila los contenidos del I Curso de Comunicación y Salud, celebrado del 9 al 12 de mayo de 2005 dentro del $8^{\circ}$ Ciclo de Comunicación de la Universidad Complutense de Madrid. En ella se describen los tres talleres simultáneos de discusión y casos prácticos que tuvieron lugar sobre campañas de publicidad de alcohol y tabaco, productos pretendidamente sanitarios juzgados por el Jurado de Autocontrol y comunicación en situaciones de crisis con posibles consecuencias en salud.

- ¿Seísmos de ida y vuelta? (2005) Rafael J. Álvarez. En Cuesta, Ubaldo., Ugarte, Aitor., Menéndez, Tania. (Coords.) Comunicación Social y Salud. Una introducción. ${ }^{40}$

\footnotetext{
${ }^{38} \mathrm{http}: / /$ eprints.ucm.es/4844/) Consultado: 10-11-2013

${ }^{39} \mathrm{http}: / / \mathrm{www}$. fundacionabbott.es/publicaciones01.html). Consultado: 12-11-2013

${ }^{40} \mathrm{http}$ ://www.madridsalud.es/publicaciones/OtrasPublicaciones/ComunicacionSaludIntr oduccion.pdf). Consultado: 2-12-2013
} 
En este capítulo de Comunicación Social y Salud. Una introducción, el autor hace un recorrido por la profesión periodística en su relación con el mundo de la ciencia. Se trata más bien de un retrato sociológico del panorama nacional del Periodismo de Salud de fechas relativamente recientes: a partir del "Informe Quiral" y centrando una gran parte de su contenido en conclusiones de este informe, les añade comentarios importantes y certeros a medida que se adentra en noticias de gran calado periodístico como las vacas locas, el aceite de orijo, el Bio Bac y el $11 \mathrm{M}$

- La información de salud en los diarios de información general (2003) Gonzalo Casino. En José María Catalán y Javier López Iglesias: ¡Infórmate en Salud! Los medios de comunicación y la información sanitaria

También en este caso se trata de un capítulo de libro (jInfórmate en Salud! Los medios de comunicación y la información sanitaria.) en el que, partiendo de la realidad actual, hace un recorrido histórico del desarrollo del Periodismo de Salud desde los años 80 incluyendo los principales temas periodísticos de salud de aquellos años y extrayendo conclusiones de todo ello:

"Los periódicos han sido a la vez motor de la educación sanitaria de la población y reflejo de la misma. El creciente espacio informativo sobre salud en los diarios españoles, es indicativo tanto del interés de la sociedad por estos temas como del creciente nivel de educación sanitaria de periodistas y lectores"

El autor añade a estas primeras consideraciones las características que observa en la presentación de la información de salud por parte de la prensa, desde la selección de las noticias, de los temas, de las fuentes, de los espacios, de los titulares y, por último y no menos importante, del profesional que se encargue de esos temas

\section{Selección de artículos publicados}

- Periodismo, salud y calidad de vida. Contenidos y Fuentes (2007) Elena Blanco Castilla y Francisco Paniagua ${ }^{41}$

Este artículo se presenta bajo varios enfoques. Considera que el campo del periodismo de salud se ha asociado principalmente a la idea de generar mensajes relacionados con enfermedades que preocupan a la población:

- Por su magnitud,

- por el riesgo de epidemia,

- por la mortalidad que conllevan

- porque sean enfermedades nuevas

Este enfoque se observa que sigue vigente, especialmente en su vertiente preventiva. Pero no es el único. Hay un segundo enfoque, que se centra en la

${ }^{41} \mathrm{http}: / /$ www.cienciared.com.ar/ra/doc.php?n=766 Consulta: 12-11-2013 
mayor atención que presta cada día el Periodismo en Salud a los temas que contribuyan a mejorar la calidad de vida de los ciudadanos

- Salud, información periodística especializada en alza (2004) Antonia González Borjas ${ }^{42}$

La autora expone el tema de la creciente demanda social de información sobre temas sanitarios y cómo esto se ha visto reflejado en los medios de comunicación, aumentando considerablemente su contenido. Plantea los problemas con los que se encuentra esta dinámica: por un lado, la falta de especialización de los periodistas $\mathrm{y}$, por otro lado, la falta de colaboración del sector sanitario. Por último, hace un análisis de una solución intermedia: la creación de gabinetes de comunicación en los servicios sanitarios como intermediarios entre las instituciones y los medios de comunicación

- Periodismo de salud en España (1999) José Luis De la Serna ${ }^{43}$

El hecho de haberse publicado en 1999 no le quita un ápice de valor a su contenido. Más bien aumenta su valor, ya que viene a analizar con antelación las tendencias más actuales: el interés de los ciudadanos por la salud, el seguimiento a través de los medios de comunicación, los resultados de las diversas encuestas del CIS, la comparación con idénticos intereses en Europa y EEUU... Y, definitivamente, su interés por el valor educativo:

"Dado que después de la escuela y la universidad, la única fuente casi tiene la población en general para continuar educándose la constituye la televisión, la radio, los periódicos e internet, la información sobre salud y medicina siempre que se haga bien- se ha convertido en un elemento potencialmente clave para ayudar a elevar la cultura científica (tan pobre en casi todas partes) y mejorar la educación para la salud de toda la sociedad"

$\mathrm{Su}$ conclusión es que, desafortunadamente, no siempre la información biomédica que llega a los medios se ajusta a la verdad, se realiza con rigor, con imparcialidad y responsabilidad, o está exenta de manipulación y de conflicto de intereses. Y, por otro lado, tampoco hay tantos periodistas especializados en comunicación y salud como se necesitarían para presentar adecuadamente los temas según la máxima "nunca escribas o hables de lo que no sabes"

- La información sanitaria en la prensa diaria (1991) Moreno Guerrero, Jorge, Martínez de las Rivas, Javier, Romero Martín, M. y Domínguez Carmona, $\mathrm{M}^{44}$

\footnotetext{
42 http://grupo.us.es/grehcco/ambitos11-12/borjas.pdf Consultado: 5-11-2013

${ }^{43} \mathrm{http} / / /$ www.cuentayrazon.org/revista/pdf/13/Num113_014.pdf Consultado: 12-112013
} 
Lo mismo sucede con este artículo publicado en 1991, pero con material investigado en 1987, precursor de los estudios que vendrían posteriormente. En él, seo los 5 diarios de mayor difusión durante 4 semanas (cada uno) de ese año. En este trabajo se analizan la extensión de las noticias, su frecuencia de aparición, qué temas aparecen y en qué áreas están distribuidas las noticias de salud. Muy importante también, es observar que en esta época, se plantea la evaluación de los mensajes sanitarios desde el punto de vista de la educación para la salud. Las conclusiones muestran:

- Que en todos los diarios aparece regularmente información sanitaria concentrada en secciones

- La que ocupa más espacio es la política sanitaria

- La más variable es la divulgación médica

- Los diarios que dedican más espacio a los sucesos, incluyen menos divulgación

- Unos pocos temas (3) concentran el 50\% de las referencias: son noticia

- Se opina que el rigor y la inteligibilidad de los contenidos de las noticias de salud. Resultan, en general, aceptables

\section{Las Asociaciones españolas de Periodismo Científico}

En unos años en los que el periodismo científico tenía poco eco entre la población, comenzaron a surgir diversas Asociaciones.

\section{Asociación Española de Comunicación Científica (AECC)}

En 1975 se fundó la Asociación Española de Comunicación Científica (AECC) $^{45}$ con el nombre de Asociación Española de Periodismo Científico (AEPC). Fundada por el periodista científico Manuel Calvo Hernando, ha trabajado los últimos 37 años en la promoción del periodismo científico en España, Europa e Iberoamérica. Sus comunicadores cubren los campos de la ciencia, la tecnología, la salud y el medio ambiente. Sus objetivos son:

- Promover la presencia de informaciones científicas en los medios de comunicación

- Estimular el reciclaje de los periodistas y comunicadores

- Velar por la independencia y la objetividad de los contenidos informativos y la libertad de expresión

- Facilitar el diálogo y las relaciones con las fuentes de información del área

- Representar a sus asociados ante organismos públicos y entidades privadas

\footnotetext{
${ }^{44}$ http://www.msssi.gob.es/biblioPublic/publicaciones/recursos_propios/resp/revista_cdr om/VOL65/65_5_445.pdf). Consultado: 10-11-2013

${ }^{45} \mathrm{http}: / /$ www.aecomunicacioncientifica.org Consulta: 8-10-2013
} 
La Asociación Española de Comunicación Científica pertenece a EUSJA (European Union of Science Journalists' Associations) y a WFSJ (World Federation of Science Journalists)

\section{Associació Catalana de Comunicació Científica (ACCC)}

En 1990 se creó en Cataluña la Asociación Catalana de Periodismo Científico, actual Associació Catalana de Comunicació Científica (ACCC) ${ }^{46}$. Se constituye como entidad profesional que agrupa a comunicadores científicos, periodistas especializados, científicos, divulgadores y editores del ámbito catalán con interés por comunicar la información científica en los medios escritos y audiovisuales

La Associació Catalana de Comunicació Científica pertenece a EUSJA (European Union of Science Journalists' Associations), a WFSJ (World Federation of Science Journalists), a COSCE (Confederación de Sociedades Científicas de España) y a FECYT (Fundación Española para la Ciencia y la Tecnología). Los principales objetivos de la ACCC son:

○ promover, difundir, ampliar y mejorar la comunicación científica en las tierras de habla catalana

- Incidir en los aspectos sociales y políticos de la información científica, velando para que contribuya al progreso social

- Facilitar la formación permanente de sus asociados

- Incentivar el debate y la autocrítica entre los comunicadores de la ciencia, la tecnología y la innovación

- Dinamizar la presencia de la ciencia en los diferentes medios de comunicación

- Facilitar la libre circulación y la divulgación de las ideas y el conocimiento científico

- Velar por la independencia y objetividad de los contenidos informativos que se generen alrededor de la ciencia, la tecnología y la innovación

- Promover la normalización de la ciencia y la divulgación científica como contenidos básicos en el ámbito de la cultura catalana

\section{Asociación Nacional de Informadores de la Salud (ANIS)}

En 1994 se creó la Asociación Nacional de Informadores de la Salud (ANIS) $^{47}$. En la Presentación de la Guía ANIS 2009 (pp.7s) incluyen su planteamiento como Asociación:

"El futuro del periodismo pasa por hacer el periodismo de fuentes, un periodismo especializado, un periodismo de información propia, un periodismo que fomenta la competencia, un periodismo que cumple una función social y un periodismo de análisis (...) un periodismo hecho por periodistas (no por ciudadanos) que orienten la agenda de la sociedad. Si no somos capaces de ofrecer algo diferente y con un punto de vista distinto de lo que cualquier

\footnotetext{
${ }^{46} \mathrm{http}$ ://www.accc.cat Consulta: 8-10-2013

${ }^{47}$ www.anisalud.com Consulta: 10-11-2013
} 
persona es capaz de contar a través de un blog o de una red social, entonces el periodismo dejará de tener su función originaria"

La ANIS y la Universidad Carlos III ${ }^{48}$ elaboraron en 2008 la "I Encuesta Nacional de Periodismo Sanitario: Una aproximación al perfil y el entorno del informador de la salud en España". En ella, los encuestados son los propios profesionales de la información de salud que opinan sobre su tarea profesional

En sus conclusiones, los encuestados se decantan por algunos temas para el futuro de la información de salud. Se trata de conseguir que, gracias a la información sanitaria, los ciudadanos tengan siempre la información adecuada para tomar las decisiones correctas en relación con su salud:

- Investigación científica relacionada con el tratamiento y curación de enfermedades

- Contenidos relacionados con salud pública

- Prevención

- Mayor difusión de contenidos acerca de la calidad de la asistencia sanitaria pública y el gasto sanitario

\section{Asociación Española de Comunicación Sanitaria_(AECS)}

A finales de 2008 se creó la Asociación Española de Comunicación Sanitaria (AECS) $^{49}$, un organismo de ámbito nacional, sin ánimo de lucro que ofrece servicios, apoyo, asesoría e información sobre comunicación en salud. La AECS es una iniciativa de profesionales en Ciencias de la Salud y Ciencias de la Comunicación interesados en las técnicas de comunicación sanitaria, tanto entre instituciones públicas y privadas como entre profesionales, medios de comunicación y población general con el objetivo de facilitar el proceso comunicativo en temas de salud

En su página web, ofrece a profesionales, instituciones, estudiantes e interesados en este campo, un punto de encuentro para diversas oportunidades de información, colaboración, asesoramiento y programas de formación, explicando su planteamiento:

"La comunicación sanitario-paciente/usuario (...) con frecuencia tropieza con dos problemas fundamentales que dificultan el proceso comunicativo; por un lado, la escasa formación poblacional en temas sanitarios y, por otro, con la no menos exigua importancia dada a las técnicas comunicativas en los desarrollos curriculares de la mayoría de las carreras profesionales sanitarias.

Por otra parte, el tratamiento de los temas sanitarios en los medios de comunicación (...) puede llegar a conseguir efectos muy lejanos a los objetivos pretendidos, bien por falta de la necesaria formación sanitaria bien por

\footnotetext{
${ }^{48}$ Autores: Antonio González Gil-García (ANIS) y María Luengo (Universidad Carlos III de Madrid)

${ }^{49} \mathrm{http} / / / \mathrm{www}$. aecs.es/ Consulta: 12-11-2013
} 
deficiente formación en técnicas de comunicación de los comunicadores. Se impone, por tanto, la creación de un foro en el que los profesionales de los medios de comunicación encargados de la comunicación sanitaria y los sanitarios en general realicen una simbiosis de sus conocimientos que permita a la población comprender con mayor facilidad aquellos temas que le permitan mejorar su calidad de vida".

Las actividades de la AECS se centran en tres secciones:

- Sección de comunicación interpersonal

- Sección de Comunicación de Masas

- Sección de Comunicación Corporativa

En general, contienen las siguientes áreas:

- Realización de programas de formación

- Organización de reuniones, actividades, congresos y otros actos formativos

- Estudios de investigación en comunicación en salud

- Realización de proyectos comunitarios

- Colaboración con otros organismos / asociaciones en comunicación sanitaria nacionales e internacionales.

- Colaboración con la Administración Pública en la realización de campañas sanitarias, y otras actividades de comunicación en salud

La Asociación edita la Revista española de Comunicación en Salud ${ }^{\mathbf{5 0}}$ desde 2010 como órgano oficial de la Asociación y en su Presentación encontramos la siguiente información:

"La RECS se dirige a la comunidad científica del sector de la Comunicación y Salud, y a aquellos que hacen uso de comunicación en salud para la toma de decisiones en materia de política sanitaria.

La RECS publica dos números anuales que contienen artículos originales de investigación experimental y teórica (..). Los títulos, resúmenes y palabrasclave de los artículos se publican también en lengua inglesa.

La RECS facilita el acceso sin restricciones a todo su contenido el cual es exclusivamente editado de forma online y se accede a través del portal de la Asociación Española de Comunicación Sanitaria (http://www.aecs.es)".

${ }^{50} \mathrm{http}: / /$ www.aecs.es/revista.html 


\section{CONCLUSIONES}

No es prolífica la investigación sobre información de salud en el periodismo escrito de la España contemporánea. Los estudios de que disponemos proceden mayoritariamente de Barcelona y Madrid, encontrándonos con algunas investigaciones en Comunidades Autónomas como es el caso de Andalucía y Galicia.

En comparación a EEUU y a los países europeos más avanzados, España ha iniciado hace poco tiempo la tarea de divulgación biomédica y de hábitos saludables. La mayoría de las investigaciones y análisis coinciden en situar el comienzo de la era moderna del Periodismo de Salud en el Estado entre finales de los años 80 y mediados de los 90, teniendo un desarrollo rápido: en menos de una década, el volumen de noticias científicas y médicas publicadas en los periódicos aumentó considerablemente.

Investigadores, Organizaciones y Asociaciones de Periodismo de Salud coinciden, junto a la Organización Mundial de la Salud, en la importancia de la formación en salud de los ciudadanos y, en consecuencia, de que los periodistas que trabajan estos temas tengan una formación especializada.

Todos ellos coinciden también en la importancia que tiene el ofrecer una información con rigor y seriedad. Por un lado, en lo que a enfermedades y riesgos de salud se refiere y, por otro lado y de forma conjunta, en lo que tiene que ver con la educación en hábitos saludables para procurar una mejor calidad de vida a la población sana.

\section{REFERENCIAS BIBLIOGRÁFICAS}

ALARCÓ HERNÁNDEZ, Antonio, MENESES FERNÁNDEZ, Ma Dolores: El Periodismo Cientifico en la prensa. Aspectos de biomedicina. Tenerife. Ecopress Comunicaciones, 2005, 233 pgs.

ALARCÓ HERNÁNDEZ, Antonio: El Periodismo Científico en prensa diaria Aspectos de biomedicina. Tesis doctoral. Dir. Ricardo Acirón Roya. Fac. de CC. de la Información. Universidad de La Laguna. (ftp://tesis.bbtk.ull.es/ccssyhum/cs80.pdf ). Consulta: 28-10-2013

ALVAREZ, Rafael. ¿Seísmos de ida y vuelta?. En Cuesta, Ubaldo; Ugarte, Aitor; Menéndez, Tania. (Coords.) Comunicación Social y Salud. Una introducción.I Curso de Comunicación y Salud. Madrid. Ed. Complutense, 2005, pp. 25-32 (http://www.madridsalud.es/publicaciones/OtrasPublicaciones/ComunicacionSa ludIntroduccion.pdf). Consultado: 2-12-2013

Asociación Catalana de Comunicación Científica (ACCC) (http://www.acc.cat). Consultado: 8-10-2013

Asociación Española de Comunicación Sanitaria (AECS) (http://www.aecs.es/).

Consultado: 12-11-2013 
Asociación Española de Comunicación Científica (http://www.aecomunicacioncientifica.org). Consultado: 21-11-2013 (AECC) Asociación Nacional de Informadores de la Salud (ANIS) (www.anisalud.com). Consultado: 10-11-2013

BARRENA, Elena. Agencias, la información en estado puro. En Catalán, José María;

LÓPEZ IGLESIAS, Javier. ;Infórmate en Salud! Los medios de comunicación y lainformación sanitaria. Madrid, Ediciones Eneida, 2003, pp. 67-78

BLANCO CASTILLA, Elena, y Paniagua, Francisco. Periodismo, salud y calidad de vida. Contenidos y Fuentes FISEC-Estrategias. Facultad de CC. Sociales de la Universidad Nacional de Lomas de Zamora. 2007, $\mathrm{n}^{\mathrm{o}}$ 8, V.2, pp. 3-24 (http://www.cienciared.com.ar/ra/doc.php?n=766). Consulta: 12-11-2013

CALVO, Manuel. Manual de periodismo científico. Barcelona, Bosch, 1997, 242 pgs.

CAMACHO MARQUINA, Idoia (Coord.). La especialización en el Periodismo. Sevilla, Comunicación Social Ed., 2010, 260 pgs.

CAMACHO MARQUINA, Idoia. Noticias sobre salud y medicina: mucho más que información En Camacho Marquina, Idoia (Coord.) La especialización en el Periodismo. Sevilla. Comunicación Social Ed., 2010, pgs. 141-161

CAMACHO MARQUINA, Idoia. La gripe A en la prensa española. En Revista Latina de Comunicación Social, 64, pp. 827-843. La Laguna (Tenerife) Universidad de la Laguna, 2009, pgs. 827-843, DOI: 10.4185/RLCS-64-2009865-827-843 (http://www.revistalatinacs.org/09/art/865_Bilbao/66_92_idoiacamacho.html). Consultado: 12-11-2013

CAMACHO MARQUINA, Idoia (IP); Ronco López, Milagros; Peñafiel Saiz, Carmen; Alazne Ayestaran Yarza y Echegaray Eizaguirre, Lázaro: La información de salud en la prensa diaria vasca (2000-2010). Investigación subvencionada por la Universidad del País Vasco/ UPV. Código EHU/10/47

CASINO, Gonzalo. La información de salud en los diarios de información general. En CATALÁN, José María.; López Iglesias, Javier (Eds.). iInfórmate en Salud! Los mediosde comunicación y la información sanitaria. Madrid: Ediciones Eneida, 2003, pp. 19-36

CATALÁN, José María y LÓPEZ IGLESIAS, Javier (Eds.). ;Infórmate en Salud!. Los medios de comunicación y la información sanitaria. Madrid, Eneida, 2003, 157 pgs.

CEAFA; AECC; FEP; CESIDA. Guía de estilo: Salud y medios de comunicación. El cáncer. Madrid, AECC, 2006, 47 pgs.

COSTA SÁNCHEZ, Carmen. Medicina y salud en la prensa. Las noticias de salud en los principales diarios de Galicia. Revista Latina de Comunicación Social, 63, Tenerife. Universidad de La Laguna, ULL, 2008, pp. 15-21. Consultado:

19-11-2013

(http://www.ull.es/publicaciones/latina/_2008/03/Costa_Sanchez.html). 
CUESTA CAMBRA, Ubaldo; Menéndez Hevia, Tania y Ugarte Iturrizaga, Aitor. (Coords.) Comunicación y Salud: Nuevos escenarios y tendencias. Madrid. Ed. Complutense, 2011, 196 pgs. (http://www.fundacionabbott.es/publicaciones01.html). Consultado: 27-112013

CUESTA CAMBRA, Ubaldo; Ugarte Iturrizaga, Aitor y Menéndez Hevia, Tania (Coords.) Comunicación y Salud: avances en modelos y estrategias de intervención. Madrid. Ed. Complutense, $2008 . \quad 185$ pgs.(http://www.fundacionabbott.es/publicaciones01.html). Consultado: 20-112013

http://www.fundacionabbott.es/Cuesta Cambra, Ubaldo; Menéndez Hevia, Tania y García Guardia, María Luisa Comunicación Social y Salud: un nuevo planteamiento estratégico. En Comunicación y Salud: avances en modelos y estrategias de intervención. Madrid. Ed. Complutense, 2008, pp. 19-39. (http://www.fundacionabbott.es/publicaciones01.html). Consultado: 10-112013

CUESTA CAMBRA, Ubaldo; UGARTE ITURRIZAGA, Aitor y MENÉNDEZ HEVIA, Tania (Coords.) Comunicación Social y Salud. Una introducción. Madrid. Ed. Complutense, 2005, 138 pgs. (http://www.fundacionabbott.es/publicaciones01.html). Consultado: 12-112013

DE LA SERNA, José Luis. Periodismo de salud en España. Revista Cuenta y Razón. $\quad \mathrm{N}^{\circ} \quad 113,1999$. (http://www.cuentayrazon.org/revista/pdf/113/Num113_014.pdfhttp://www.cue ntayrazon.org/revista/pdf/13/Num113_014.pdf)

Consultado: 12-11-2013

DE SEMIR. Vladimir. Periodismo científico: España es diferente. En I

Congreso Nacional de Periodismo Científico. Madrid. Consejo Superior de Investigaciones

Científicas, 1990, pp. 175-183.

GONZÁLEZ BORJAS, Antonia. Salud, información periodística especializada en alza. ÁMBITOS, Revista Andaluza de Comunicación. Sevilla, $\mathrm{n}^{\circ} 11-12,1^{\circ}$ y $2^{\circ}$ semestre, 2004, pp.301-310. (http://grupo.us.es/grehcco/ambitos1112/borjas.pdf) Consultado: 5-11-2013

HERNÁNDEZ GARCÍA, L. Análisis de los contenidos sociosanitarios en prensa local. Revista Española de Comunicación en Salud. Vol 2, no 1, 2011, pp. 2431. (http://www.aecs.es/revista_1_2.html) Consultado: 15-11-2013.

Instituto de Información Sanitaria. Barómetro Sanitario, 2009, 2010, 2011 y 2012. Madrid: Ministerio de Sanidad, Política Social e Igualdad:

Barómetro Sanitario 2012 y tabla comparativa por Comunidades 1995-2012: 
http://www.msc.es/estadEstudios/estadisticas/docs/BS_2011_total_marginales.pdf https://www.msssi.gob.es/estadEstudios/estadisticas/sisInfSanSNS/informeAnu al2012.htm

Consultado: 12-9-2013

Barómetro Sanitario 2011:

http://www.msc.es/estadEstudios/estadisticas/docs/BS_2011_total_marginales.pdf http://www.mspsi.gob.es/estadEstudios/estadisticas/sisInfSanSNS/informeAnual.htm Consultado: 12-9-2013

Barómetros Sanitarios 2009-2010:

www.msps.es/estadEstudios/estadisticas/inforRecopilaciones/barometro/home.htm Consultado: 12-9-2013

Instituto Internacional de Comunicación y Salud (INICyS)

(www.inicys.com/). Consultado: 11-9-2013

JURADO SALVÁN, Enrique. Evolución de la información sanitaria en la prensa escrita durante la Transición española. Tesis doctoral. Dir.Javier FERNÁNDEZ DEL MORAL. Dpto. Periodismo II. Fac. de CC de la Información. Universidad Complutense de Madrid, 2003 (http://eprints.ucm.es/4844/) Consultado: 10-11-2013

JURADO SALVÁN, Enrique. Evolución de la información sanitaria en la prensa escrita durante la Transición española (1977-2002). Madrid. Ed. Complutense, 2005, 419 pgs.

LARROSA, Coral. En CATALÁN, José María.; López Iglesias, Javier (Eds.): ¡Infórmate en Salud! Los medios de comunicación y la información sanitaria. Madrid: Ediciones Eneida, 2003, pp.37-52

LEÓN VERGARA, María .Reyes; HERRERA USAGRE, Manuel y DELGADO RAMOS, Rosario .María. .Calidad sanitaria y Mass Media. El impacto mediático de la localización de notas de prensa sobre acreditación sanitaria en Andalucía. Revista de Comunicación y Salud, Madrid, Ed. INICyS. Vol 2, nº 1 , 2012, pp. 5-17.

(www.revistadecomunicacionysalud.org/index.php/rcys/search/titles) Consulta; 5-11-2013

MÁRQUEZ HERNÁNDEZ, Verónica. Los contenidos sanitarios en la prensa nacional. Revista Española de Comunicación en Salud. Ed. Asociación Española de Comunicación Sanitaria. Vol 1, $\mathrm{n}^{\mathrm{o}}$ 2, 2010, pp. 164-171. http://www.aecs.es Consultado: 8-11-2013

MARTÍNEZ SEGURA, Pablo. Medios de comunicación y salud En Cabasés, Juan; Villalví, Joan Ramón y Aibar, Carlos (Eds.). Informe SESPAS 2002: Invertir para la salud. Prioridades en salud pública. Madrid. Ed. Sociedad Española de Salud Pública y Administración Sanitaria. Cap. 28, 2002, pp. 581-598 (http://www.sespas.es/informes.php). Consultado: 5-10-2013 
MORENO GUERRERO, Jorge; Martínez de las Rivas Maroto, Javier; Romero Martín, M. y Domínguez Carmona, M. La información sanitaria en la prensa diaria. Revista Española de Salud Pública, nº 5, 65, septiembre-octubre, 1991, pp. $445-450$

(http://www.msssi.gob.es/biblioPublic/publicaciones/recursos_propios/resp/revi sta_cdrom/VOL65/65_5_445.pdf). Consultado: 10-11-2013

Observatorio de Comunicación y Salud (OCS). (www.portalcomunicacion.com/ocs/). Consultado: 9-12-2013

Observatorio de la Comunicación Científica / Fundación Vilá Casas. Informe

Quiral: años 2000-2013.- (http://www.fundaciovilacasas.com/es/saludiinforme-quiral/) Consultado: 6-10-2013.

Observatorio de la Comunicación Científica / Fundación Vilá Casas. Informe Quiral: años $1997-2006 .-\quad 68$ pgs. (www.upf.edu/pcstacademy/_docs/Quiral10.pdf).Consultado: 6-10-2013

Organización Mundial de la Salud (OMS): Informes de Estadísticas Sanitarias Mundiales 2005-2013. Observatorio Mundial de la Salud (GHO). (http://www.who.int/gho/publications/world_health_statistics/en/index.html). Consultado: 5-12-2013

Organización Mundial de la Salud (OMS): Informes sobre la salud en el mundo 1995-2013 . ( http://www.who.int/whr/es/index.html). Consultado: 5-12-2013

Organización Mundial de la Salud (OMS) : Proyecto de $12^{\circ}$ Programa General de Trabajo. Proyecto para examen por los Comités Regionales en 2012 (Período 2014-19).

(http://apps.who.int/gb/s/s_wha65.htmhttp://apps.who.int/gb/ebwha/pdf_files/W HA65/A65 5Add1sp.pdfhttp://apps.who.int/gb/ebwha/pdf_files/WHA66/A66_6Add1-sp.pdf). Consultado: 5-12-2013

Organización Mundial de la Salud (OMS): Determinantes sociales de la salud. Resolución WHA62/R14 de la Asamblea Mundial de la Salud (2005-2008). (http://www.who.int/social_determinants/thecommission/es/). Consultado: 12$11-2013$

Organización Mundial de la Salud (OMS). Glosario de Promoción de la salud. 1998

(http://www.msc.es/profesionales/saludPublica/prevPromocion/promocion/glos ario/home.htm). Consultado: 5-12-2013 
Organización Mundial de la Salud OMS). Proyecto para examen por los Comités Regionales para el período 2014-2019, presentado en mayo de 2012 a la $65^{\mathrm{a}}$ Asamblea Mundial de la Salud y al Consejo Ejecutivo en su 131 a reunión. (http://www.who.int/about/resources_planning/gpw_for_reg_commitees_2012_ es.pdf) Consulta 12-10-2013

REVUELTA, Gemma; DE SEMIR, Vladimir; Armengol, Clara y Gian Franco Selgas.-Informe Quiral 2011.Cáncer. Barcelona, Fundació Vilá Casas y Observatorio de la Comunicación Científica de la UPF, 2012.

(http://www.fundaciovilacasas.com/ca/salut-informe-quiral/informe-quiral-2011cancer/ pub:60/). Consultado: 15-9-2013

REVUELTA, Gemma y DE SEMIR, Vladimir (Dirs.): Informe Quiral 2009. Medicina, Comunicación y Sociedad. Barcelona. Fundació Vilá Casas y Observatorio de la Comunicación Científica de la UPF, 2010. 88 pgs. Consultado: 10-10-2013

(http://www.fundaciovilacasas.com/ca/salut-informe-quiral/informe-quiral-2009)

REVUELTA, GEMMA Y De Semir, Vladimir (Dirs.): Informe Quiral 1997-2006. Medicina y salud en la prensa diaria.. Fundació Vilá Casas y Observatorio de la Comunicación Científica de la Universitat Pompeu Fabra. Barcelona, 2007. http://www.upf.edu/pcstacademy/_docs/Quiral10.pdfhttp://www.upf.edu/pcstacade my/_docs/Quiral10.pdf). Consultado: 30-10-2013

REVUELTA, Gemma. y MINELLI DE OLIVEIRA Janaina.. La salud y la biomedicina en la prensa diaria. Un análisis de diez años. . Periodística: Revista Académica. Vol. No 11, 2008, pp. 55-67.

(http://www.raco.cat/index.php/Periodistica/article/view/245697) Consultado: 8$11-2013$

Seminario de Comunicación y Salud de la Universidad de Navarra. Facultad de Comunicación de la UN e Instituto Novartis de Comunicación en Biomedicina (INCB). 10 Edición: Comunicación y Salud: Hacia la convergencia de una extraña pareja. Pamplona. 2013 (http://www.novartis.es/sala-deprensa/comunicados-de-prenssa/2013/01/21). Consultado: 2-11-2013

Sociedad Española de Medicina Familiar y Comunitaria (SEMFyC): Grupos de Trabajo sobre Comunicación y Salud.(http://comunicacionysalud.es/). Consultado: 15-11-2013

UGARTE ITURRIZAGA, Aitor; Menéndez Hevia, Tania y Cuesta Cambra, Ubaldo (Coords.) Prensa escrita y salud en Madrid. Una revisión del modelo de la Agenda Setting y su aplicación a la dinámica de las noticias sobre salud en Madrid. Madrid. Ed. Complutense, 2009, 136 pgs.. (http://www.fundacionabbott.es/publicaciones01.html). Consultado: 20-112013 
UGARTE ITURRIZAGA, Aitor. El binomio comunicación y salud: Relaciones e investigación En Cuesta Cambra, Ubaldo; Ugarte Iturrizaga, Aitor y Menéndez Hevia, Tania (Coords.): Comunicación y Salud: avances en modelos y estrategias de intervención. Madrid. Ed. Complutense. 2008, pgs. 39-48

http://www.fundacionabbott.es/publicaciones01.html). Consultado: 2-11-2013. 